\title{
Comparing Two Models of Large-Scale White Sea Hydrodynamics and Thermal Dynamics
}

\author{
Ilya Chernov, Alexey Tolstikov \\ 1 - Institute of Applied Mathematical Research KRC RAS; Petrozavodsk State University \\ 2 - Northern Water Problems Institute KRC RAS. Address: 185910, IAMR KRC RAS, \\ Pushkinskaya 11, Petrozavodsk, Russia
}

\begin{abstract}
To calculate the water temperature of the White Sea we used two models of large-scale hydro- and thermal dynamics, maintained by the authors. Comparing two models, we show that the first one describes summer hydrophysical conditions better, while the second model is better for winter conditions. Now we are trying to improve and combine two models in order to describe the state of the Sea more accurately.
\end{abstract}

Keywords - large-scale sea models, the White Sea.

\section{INTRODUCTION}

The White sea has some unique properties: tidal flows dominate; the sea is shallow and thus bathymetry is important; high density of available potential density and potential density; significant stratification. This makes the sea an interesting oceanological object [1]. Mathematical and computer modeling allows getting maximal amount of information from data, reduce costs, estimate quantities that are difficult or impossible to measure, make a forecast, estimate influence of different factor, and perform numerical experiment (which is possible when natural experiment is hardly possible).

Intensive development of computers in the recent decades significantly enforced the power of mathematical modeling. Beside ocean modeling and simulation [2], complex models of the White sea were developed. The book [1] reviews the White sea research, including numerical modeling and simulation. Nonlinear interaction of dynamical processes is very important in this sea; this implies need for complex hydrodynamical models of high resolution and demand efficient algorithms and highperformance computers. Dominating tidal flows facilitate modeling, making initial data less important.

\section{THE MODELS}

In this paper we describe and compare two models of thermal and hydrodynamics of the White sea. The first model was developed by I.A. Neelov (see [1] and references therein), the second is the model of the Arctic ocean created by N.G. Iakovlev [3] in the Institute of Numerical Mathematics (Moscow) adapted to the White sea by the authors. Both models are based on primitive equations in spherical coordinates with free surface and the Boussinesq approximation. Vertical grid is inhomogenous and consists of 22 and 16 levels. Spatial steps of the models are 3 ' and $4^{\prime}$ of latitude and $6^{\prime}$ and 14 of longitude. Time steps are $10 \mathrm{~min}$ and $6 \mathrm{~min}$, respectfully. Both models are climate models with seasonal components. Tide is described as oscillation of the sea level at the White sea-Barents sea liquid boundary. Atmospheric forcing (precipitation, clouds, air temperature, pressure, and humidity) are taken from the NCEP data [5] with linear interpolation both in space and time. Wind is either taken from the NCEP data or calculated via air pressure as quazi-geostrophical. Runoff of main rivers is taken into consideration: rivers are described as fresh water bays with given normal water velocity at the liquid boundary (calculated via mean yearly runoff with empirical month distribution) and monthly-mean temperature. The main difference between the models is the description of the sea ice, also some parametrizations, discretization, and details of calculation algorithms differ. Since 2012 both models are remotely available via Internet at the computer cluster of the Karelian Research Centre [6] in multiuser mode. The cluster consists of 10 nodes with two 4-core processors and a control node, the peak performance is 851 Gflops. The BOINC-based desktop grid is available. It is convenient for solving multiple poorly connected problems (e.g. estimating reaction of the sea to different model forcing).

\section{RESULTS AND DISCUSSION}

Both models show almost the same behavior of water temperature. In. Fig. 1 there are monthly-mean water temperatures averaged over the sea surface for the two models. The second models gives higher surface temperature; though, general behavior is represented in the similar way. The lowest temperatures were in 1969 and 1978, the highest ones were in 1972 and 1989.

Fig. 2 and 3 compare distribution of surface temperature at some time (after 30 model years) for the two models. 


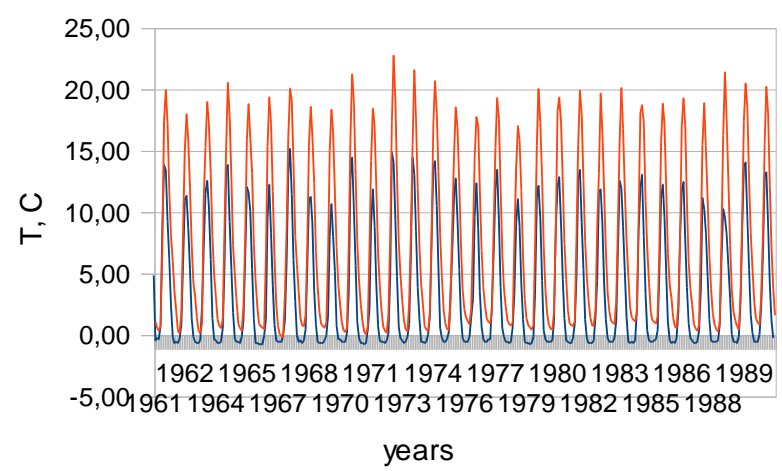

Fig. 1. Monthly-mean sea surface water temperature for the two models (higher peaks are for the first model, lower ones are for the second).

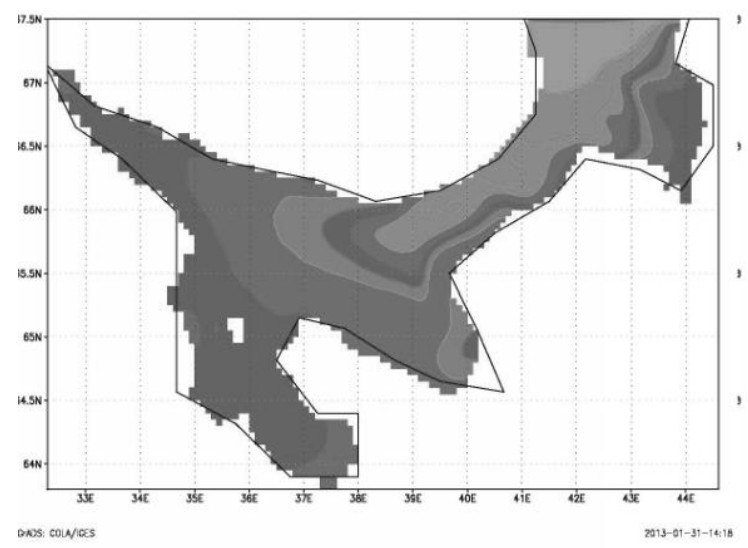

Fig. 2. Surface temperature distribution, 30 model years calculated, model 1.

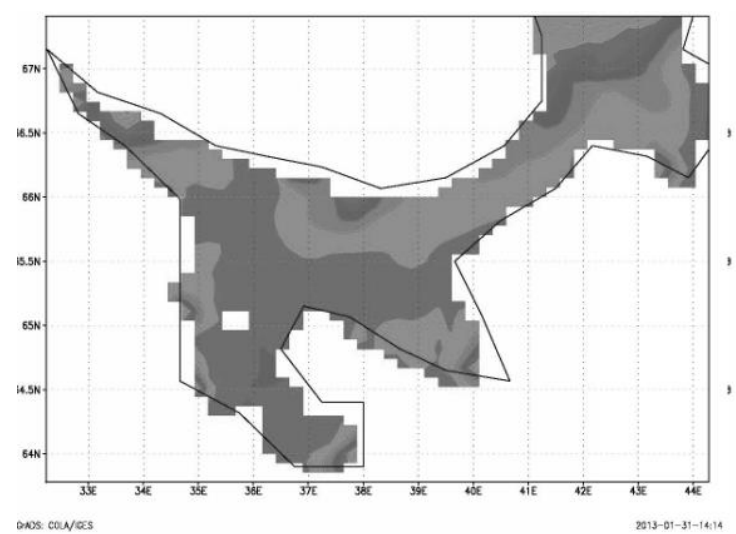

Fig. 3. Surface temperature distribution, 30 model years calculated, model 2.

Despite some difference, both models represent fronts in bays, the Kelvin wave, higher temperature of water in shallow bays. The same is true also for salinity; fig. 4 and 5 compare distribution of surface salinity for the two models. The Kelvin wave, and freshening effect of rivers are clearly represented.

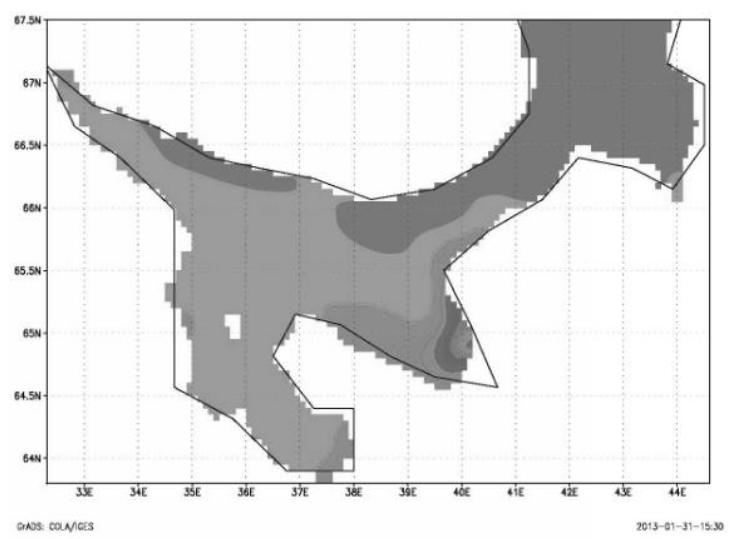

Fig. 4. Surface salinity distribution, 30 model years calculated, model 1.

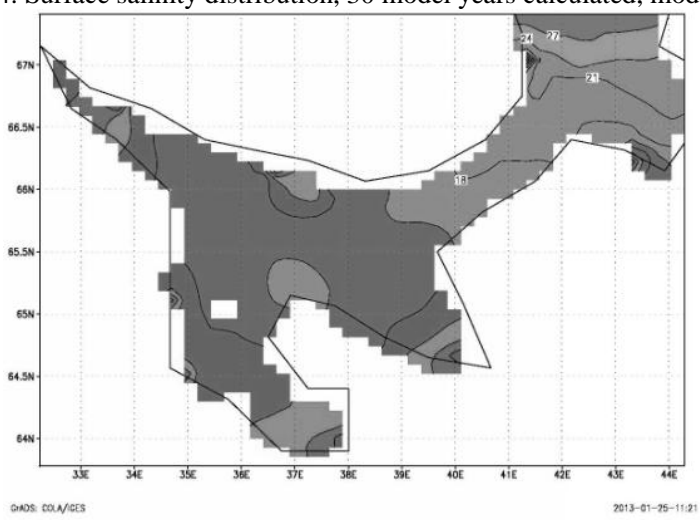

Fig. 5. Surface salinity distribution, 30 model years calculated, model 2.

The structure of vertical distribution of temperature and salinity also corresponds to observations: mixed layer near the surface, thermocline (or halocline), and almost constant temperature (or salinity) in deep places. Both models represent qualitative properties of other largescale hydrodynamical fields: ice velocity, area, and compactness (although thicker, than real, ice appears in November and is out by July).

\section{NUMERICAL EXPERIMENT}

The strong side of climatic models is their ability to perform numerical experiments, including those that are hardly possible or absolutely impossible. As an example, let us consider the influence of air temperature on the sea system. In. fig. 6 there are curve of time-dependent water temperature averaged over the sea surface. One can see that the influence is not drastic: the average temperature grows to $1,27^{\circ}$ during 60 years, while the increase of the maximal temperature is only $2^{\circ}$. Minimal temperature does not change, because it is equal the melting temperature independent on the atmosphere. This conclusion is also justified by fig. 7-9 showing the surface temperature distribution similar to fig. 3 but after 30 years of real air temperature, warmer $\left(+1^{\circ}\right)$ and colder $\left(-1^{\circ}\right)$ atmosphere. The structure of the temperature field remains almost unchanged. Temperature change is about $1^{\circ} \mathrm{C}$ which is comparable to the change of the air temperature. 


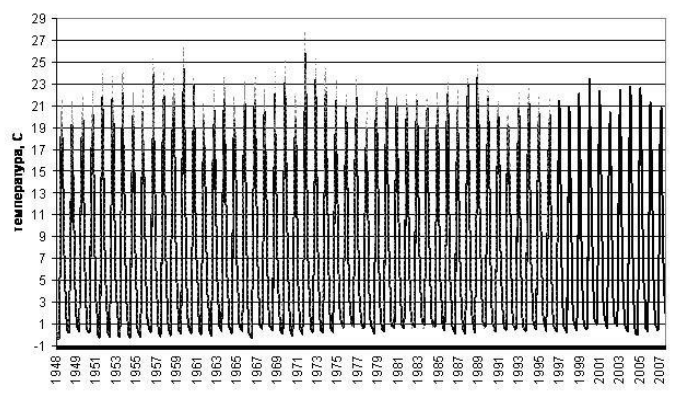

Fig. 6. Average surface water temperature for real atmospheric conditions and modified $(+30 \mathrm{C}$ added $)$ air temperature.

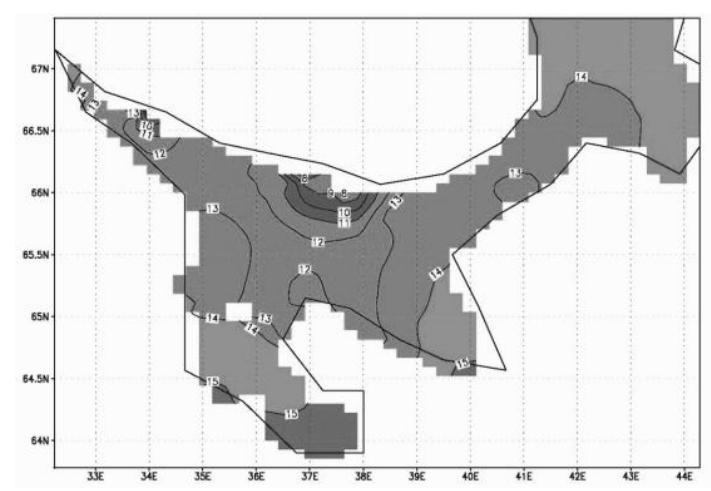

Fig. 7. Surface temperature distribution, 30 years of real atmospheric forcing (NCEP data).

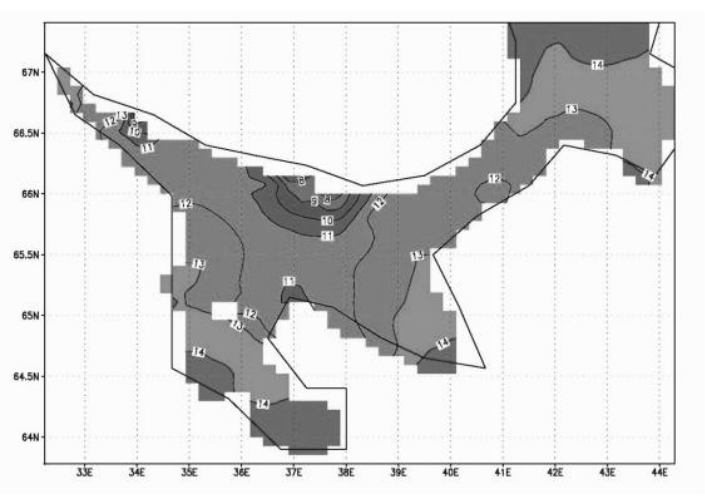

Fig. 8. Surface temperature distribution, 30 years of warmer $\left(+1^{\circ}\right)$ air.

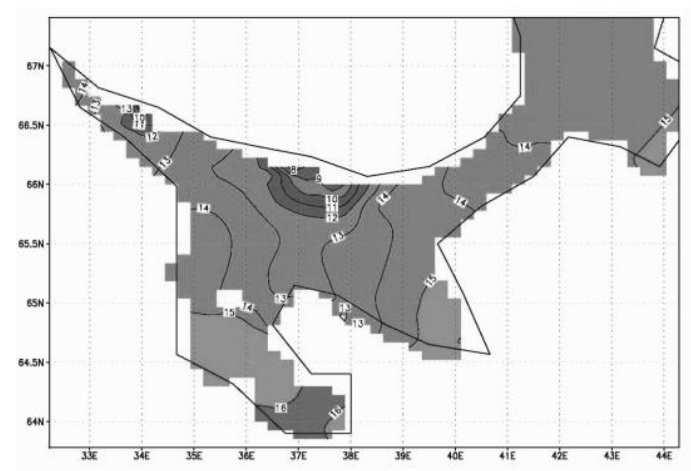

Fig. 9. Surface temperature distribution, 30 years of colder $\left(-1^{\circ}\right)$ air.
Similar influence is also at the salinity; fig. 10-12 show the surface salinity distribution for the same cases. Salinity even does not become higher or lower, just isohalines change slightly. General conclusion is that lower air temperature helps salinity to propagate from the Barents sea. Current velocity and ice distribution also react slightly on the air temperature change. Both models represent main circulations in the Basin and in bays and the Ekman spiral.

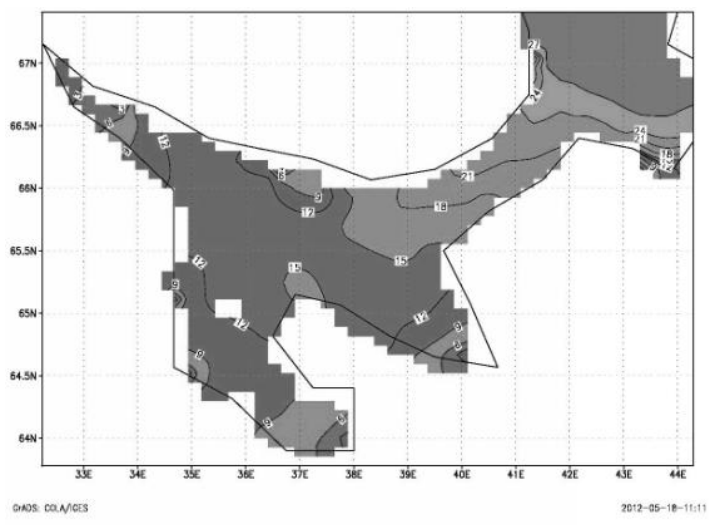

Fig. 10. Surface salinity distribution, 30 years of real atmospheric forcing (NCEP data).

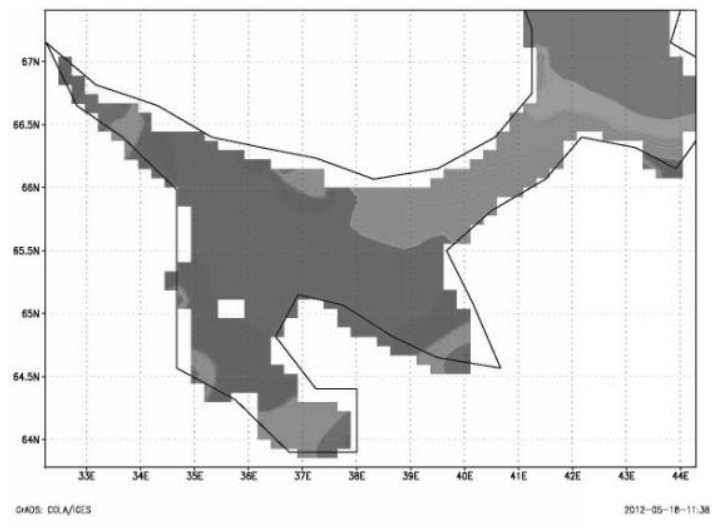

Fig. 11. Surface salinity distribution, 30 years of warmer $\left(+1^{\circ}\right)$ air.

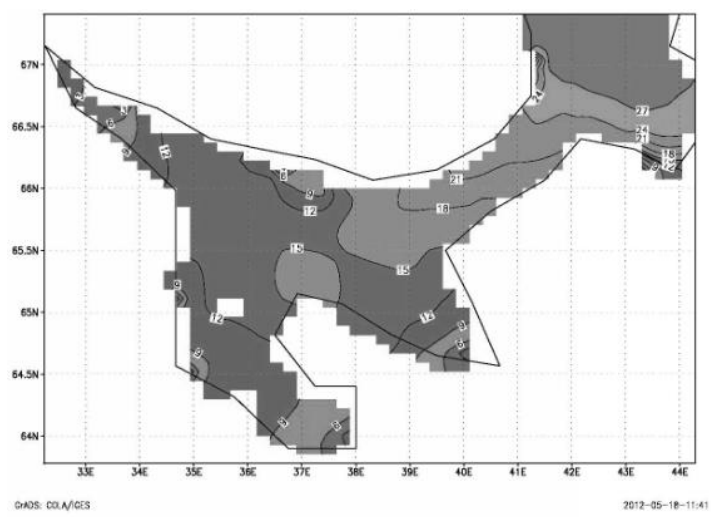

Fig. 12. Surface salinity distribution, 30 years of colder $\left(-1^{\circ}\right)$ air.

\section{$\mathrm{V}$ CONCLUSION}

Both models represent main qualitative properties of large-scale hydrodynamical and thermodynamical 
fields of the White sea. We currently are working on adjusting parameters in order to improve the quality of approximation of the measurements and the model data. The first models seems to give better results for the iceless parts of the year, while the second is better for the winter time. Beside parametrizations, there are other difficulties. Little is know about, for example, water temperature and salinity with respect to time, depth, and the point at the surface at the liquid boundary between two seas. These data serve as boundary conditions; we are planning to include part of the Barents sea into the domain in order to, firstly, use data measured there and, secondly, to reduce the influence of the boundary data on the sea. This is going to be useful also for ice dynamics, because ice gone out of the domain is lost and is does not come back in case when the current change its direction (while it must, and the situation is typical for the White sea with its strong induced tides). The general conclusion is that both models are useful tools for investigating largescale dynamics of the White sea.

\section{ACKNOWLEDGMENTS}

This study was partly financially supported by the Program of strategic development of Petrozavodsk State University, within measures on improvement of scientific research activity.

\section{REFERENCES}

[1] N. Filatov, D. Pozdnyakov D., O.M. Johannessen, L.H. Pettersson, and L.P. Bobylev, White sea, its Marine Environment and Ecosystem Dynamics Influenced by Global Change. SpringerPraxis, 2005.

[2] A.S. Sarkisyan, "On some achievements and major problems in mathematical modeling of climatic characteristics of the Ocean (critical analysis)", Izvestiya, Atmospheric and Oceanic Physics, vol. 46, iss. 6, pp. 668-676, Dec. 2010.

[3] N.G. Yakovlev, "Coupled Model of Ocean General Circulation and SeaIce Evolution in the Arctic Ocean", Izvestiya, Atmospheric and Oceanic Physics, vol. 39, 3, pp. 355-368, 2003.

[4] Park S., Choi K.S., Joe K.S., Kim W.H., Kim H.S. Variations of landfill leachate properties in conjunction with the treatment process. Environ. Technol., 22. 2001, pp. 639-645.

[5] Kalnay et al.,"The NCEP/NCAR 40-year reanalysis project", Bull. Amer. Meteor. Soc., vol. 77, pp. 437-470, 1996.

[6] Centre for Collective Use of the Karelian Research Centre [Online]. Available: http://cluster.krc.karelia.ru [Accessed $28 \mathrm{Feb}$. 2013]. 